\title{
Promotion of microglial phagocytosis by tuftsin stimulates remyelination in experimental autoimmune encephalomyelitis
}

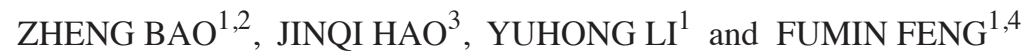 \\ ${ }^{1}$ School of Public Health, North China University of Science and Technology, Tangshan, Hebei 063210; \\ ${ }^{2}$ Child Health Division, Tongzhou Maternal and Child Health Hospital of Beijing, Beijing 101101; \\ ${ }^{3}$ School of Public Health, BaoTou Medical College, Baotou, Neimenggu 014040; ${ }^{4}$ Center-Laboratory, \\ North China University of Science and Technology, Tangshan, Hebei 063210, P.R. China
}

Received March 23, 2019; Accepted October 2, 2019

DOI: $10.3892 / \mathrm{mmr} .2019 .10788$

\begin{abstract}
Microglia were once thought to serve a pathogenic role in demyelinating diseases, particularly in multiple sclerosis (MS). However, it has recently been shown that in the experimental autoimmune encephalomyelitis (EAE) model of MS, microglia could serve a protective role by promoting remyelination via the efficient removal of apoptotic cells, the phagocytosis of debris and the support of myelinating oligodendrocytes. The aim of the present study was to determine if the effect of microglia could promote the recovery of EAE and attenuate symptoms in EAE. The severity of EAE was assessed by clinical scores, pathologic changes revealed by luxol fast blue staining and immunohistochemical techniques. The results suggested that microglia reduced clinical scores in mice, suppressed ongoing severe EAE and promoted remyelination and recovery in EAE mice. In addition, following induction with tuftsin, the M1/M2 cytokine balance was shifted, downregulating the proinflammatory M1 response and upregulating the anti-inflammatory M2 response. Generally, microglia can stimulate remyelination, which serves a protective role in different phases of EAE and may represent a potential therapeutic strategy for the treatment of MS.
\end{abstract}

\section{Introduction}

Microglial cells are the resident immune cells of the central nervous system (CNS) and can quickly respond to CNS injury; activation occurs rapidly within hours (1). Once the microglia are activated, they can secrete and release cytokines and chemokines, such as interleukin (IL)-1, IL-2, IL-6, transforming growth factor (TGF) 131, and matrix metalloproteinase (MMP)9, and express

Correspondence to: Dr Fumin Feng, Center-Laboratory, North China University of Science and Technology, 21 Bohai Road, Tangshan, Hebei 063210, P.R. China

E-mail: hblgffm@126.com

Key words: microglia, multiple sclerosis, experimental autoimmune, encephalomyelitis, remyelination, tuftsin complement components and their receptors (2). Traditionally, microglial cell activity in the CNS has been considered detrimental, as they recruit and reactivate T cells in the CNS by secreting MMPs, disrupting the blood-brain barrier, upregulate major histocompatibility complex II molecules and exert OPC toxicity by producing inflammatory cytokines and reactive oxygen species (3). Microglia have been reported to participate in the pathogenesis of many human diseases, such as Alzheimer's disease, multiple sclerosis (MS), autoimmune diseases and other disorders (4). Particularly in MS, microglia have been reported to serve dual roles in demyelination in the CNS, including protective and pathogenic roles (5). However, previous studies have demonstrated that microglia may serve an important role in the remyelination process, through phagocytosing debris from cells and destroyed myelin, supporting myelinating oligodendrocytes and promoting axonal regeneration $(6,7)$.

Tuftsin (Thr-Lys-Pro-Arg) was first described by Fridkin et al (8) and was thought to be a phagocytosis-stimulating factor for cells of monocytic origin. Tuftsin activates macrophages through binding to receptors that are expressed by cells of monocytic origin, including microglia (9).

The present study investigated the role of microglia in EAE progression. Tuftsin was used to alter the activation of microglia. Early administration of tuftsin altered the activation of microglia and attenuated the humoral immune responses associated with EAE progression to different degrees.

\section{Materials and methods}

Experimental animals. Adult (8-9 weeks old and 18-20 g) female C57BL/6 mice were purchased from North China University of Science and Technology. All mice were housed in a temperature-controlled room under a 12-h light/dark cycle for 4 weeks with food and water ad libitum. The mice were randomly divided into three groups; control group $(n=12)$, experimental autoimmune encephalomyelitis group (EAE group, $n=12)$ and Tuftsin group $(n=12)$. All protocols were approved by the Animal Ethics Committee of the North China University of Science and Technology.

Induction of EAE in mice. EAE mice were induced with MOG35-55 $(200 \mu \mathrm{g})$, and the mice were intraperitoneally 
injected with pertussis toxin (500 $\mathrm{ng}$, List Biological Laboratories, Inc.) at 0 and $48 \mathrm{~h}$ following immunization. At least two investigators weighed and evaluated the animals for clinical scores in a blinded manner. There were two animals which appeared to be in intolerable distress and self-mutilated limbs; these were sacrificed using pentobarbital sodium $(150 \mathrm{mg} / \mathrm{kg})$.

Clinical assessment. Clinical scores (10) were determined in accordance with the following criteria: 0 , healthy; 1 , limp tail; 2, ataxia and/or paresis of the hind limbs; 3, paralysis of the hind limbs; 4, paresis and/or paralysis of the forelimbs; 5 , moribund or dead.

Time-controlled drug delivery. The present study used ALZET mini-osmotic pumps to control drug delivery over time. The mice were injected with either PBS or $500 \mathrm{mM}$ tuftsin [Gen Script (Nanjing) Co., Ltd.] at a rate of $0.25 \mathrm{ml} / \mathrm{h}$ (total volume was $100 \mathrm{ml}$ ). Pumps were implanted subcutaneously in the backs of anaesthetized mice on day 1 following immunization. On day 15 , the pumps were replaced with fresh pumps and maintained thus until day 28 .

Histological staining and immunohistochemistry. Spinal cords were obtained from anaesthetized mice, which were perfused intracardially with $4 \%$ paraformaldehyde. The samples underwent a dehydration in graded ethanol $(70 \%$ ethanol 3-5 min; 80\% ethanol 3-5 min; 90\% ethanol 3-5 min; 95\% ethanol 3-5 min). Paraffin-embedded tissue sections were cut in the coronal plane at a thickness of $5 \mu \mathrm{m}$. Histological staining, including LFB staining, was performed to identify demyelination. The sections were left in LFB solution (Beijing Solarbio Science \& Technology Co., Ltd.) at $56^{\circ} \mathrm{C}$ overnight, excess stain rinsed off with $95 \%$ ethyl alcohol and distilled water, differentiated in lithium carbonate solution for $30 \mathrm{sec}$ and $70 \%$ ethyl alcohol for $30 \mathrm{sec}$, counterstained in cresyl violet solution (Guidechem) for 30-40 sec, rinsed in distilled water, differentiated in $95 \%$ ethyl alcohol for $5 \mathrm{~min}$ then placed in $100 \%$ alcohol for 5 min (twice) and finally two baths in xylene for 5 min each. Immunohistochemistry was performed with anti-myelin basic protein (MBP) antibodies to identify MBP (1:100; sc-271524, Santa Cruz Biotechnology, Inc.). Hematoxylin was used to stain cell morphology. The sections were observed under light microscope (magnification, x40) (11) and analyzed by Image 2 Pro plus 5.0 (Media Cybernetics, Inc.).

Reverse transcription-quantitative $(R T-q) P C R$. Total RNA was extracted from the brain and spinal cord in all groups using the RNAeasy Micro kit purchased from OMEGA Company following the manufacturer's instructions. Reverse transcription was performed on $1 \mu \mathrm{g}$ of total RNA with an RT-PCR kit (Life Technologies; Thermo Fisher Scientific, Inc.), Purity quantification, cDNA synthesis and qPCR were performed according to the manufacturer's protocols. Reaction procedures were as follows: An initial step at $95^{\circ} \mathrm{C}$ for $5 \mathrm{~min}$, 40 cycles of $94^{\circ} \mathrm{C}$ for $15 \mathrm{sec}$, and $60^{\circ} \mathrm{C}$ for $34 \mathrm{sec}$; reaction volume $20 \mu \mathrm{l}$. The primer sequences were: GAPDH: Forward: 5'-TTCACCACCATGGAGAAGGC-3', Reverse: 5'-GGCATG GACTGTGGTCATGA-3'; tumor necrosis factor (TNF)- $\alpha$ :
Forward: 5'-CATCTTCTCAAA ATTCGAGTGACAA-3', Reverse: 5'-TGGG-AGTAGACAAGGTACAACCC-3'; IL-10: Forward: 5'-TGGCCACACTTGA-GAGCTGC-3', Reverse: 5'-TTCAGGGATGAAGCGGCTGG-3'; TGF- $\beta$ : Forward: 5'-CCGCAACAACGCAATCTATG-3', Reverse: 5'-AGCCCT GTATTCCGTCTCCTT-3'.

qPCR was carried out using SYBR green mix (Roche, USA) and analyzed using the $2^{-\Delta \Delta C q}$ method (11). The relative expression levels of the mRNAs were reported as fold changes vs. control.

Western blot analysis. Protein was extracted from the brain and spinal cord. The samples were lysed in Tissue Protein Lysis Solution (Thermo Fisher Scientific, Inc.). The protein concentration was determined using a BCA protein assay kit (OriGene Technologies, Inc.) and $25 \mu \mathrm{g}$ of protein was loaded per lane. The primary antibodies used were specific for $\beta$-actin (1:1,000; cat. no. AB8227, Abcam), for the detection of ionized calcium binding adaptor molecule 1 (iba-1; 1:1,000; no. AB5076, Abcam), and for the detection of MBP (1:500, no. sc-271524, Santa Cruz Biotechnology, Inc.). Protein extracts were separated by electrophoresis on $12 \%$ SDS-PAGE gels and transferred onto polyvinylidene fluoride membranes. The membranes were blocked in 5\% non-fat milk for $2 \mathrm{~h}$ at room temperature and washed three times in PBS with Tween-20 $(0.05 \%)$. The membranes were sequentially incubated at $4^{\circ} \mathrm{C}$ with primary antibodies $(2 \mathrm{~h})$ and secondary antibodies $(1 \mathrm{~h}$; 1:1,000; no. AB205718, Abcam) and enhanced chemiluminescence (ECL) solution. Finally, the images were captured and analyzed by using ImageJ v1.41 software (National Institutes of Health).

Statistical analysis. Data from experiments performed in triplicate were analyzed by One-way ANOVA, and multiple comparisons between groups were performed using the Student-Newman-Keuls method. The results are shown as the mean \pm standard error of mean. $\mathrm{P}<0.05,{ }^{* *} \mathrm{P}<0.01$ or ${ }^{* * *} \mathrm{P}<0.001$ was considered to indicate a statistically significant difference.

\section{Results}

Effect of tuftsin on the activation of microglia in EAE. To explore the function of microglia in EAE, the expression of microglia in mice induced with tuftsin or PBS was first examined. EAE mice were induced with the MOG35-55 peptide. The expression levels of all groups were compared using western blotting at different time points of EAE. As shown in Fig. 1, western blots indicated increases in iba- 1 and MBP expression in the spinal cords of tuftsin-treated mice compared with those of the EAE mice on day $7(\mathrm{P}>0.05)$, day $14(\mathrm{P}<0.01)$, day 21 $(\mathrm{P}<0.01)$, and day $28(\mathrm{P}<0.01)$. In a comparison of the EAE group and the control group, expression of iba-1 in EAE group demonstrated notably higher expression than the control group on day $14(\mathrm{P}<0.01)$, day $21(\mathrm{P}<0.001)$, and day $28(\mathrm{P}<0.001)$; the EAE group demonstrated significantly higher expression of MBP compared with the control group on day $14(\mathrm{P}<0.001)$, day $21(\mathrm{P}<0.001)$ and day $28(\mathrm{P}<0.001)$.

Effect of tuftsin on EAE clinical scores. Clinical scores are one of the validated behavioral methods used to evaluate 
A

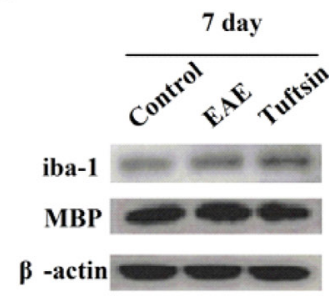

C
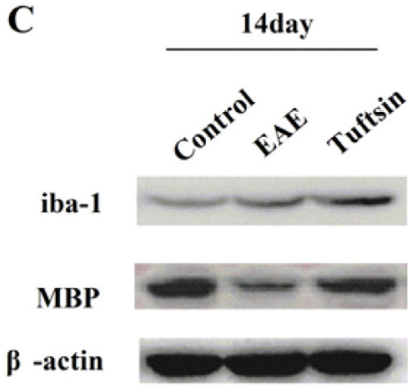

E

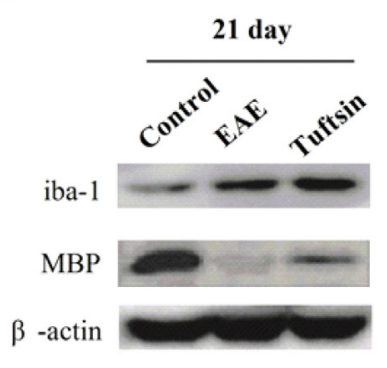

G

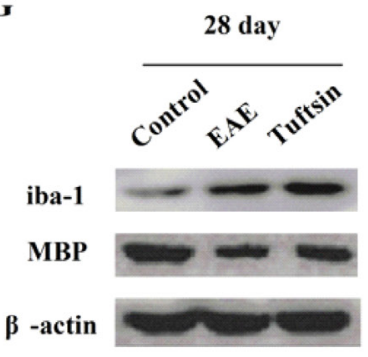

B

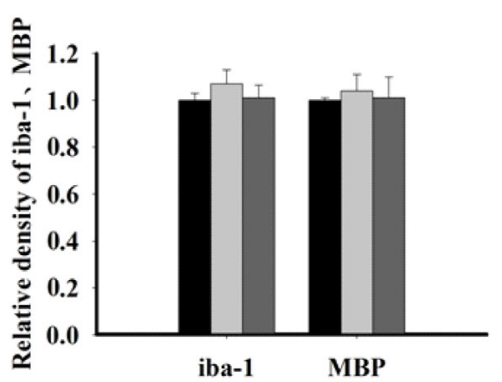

D

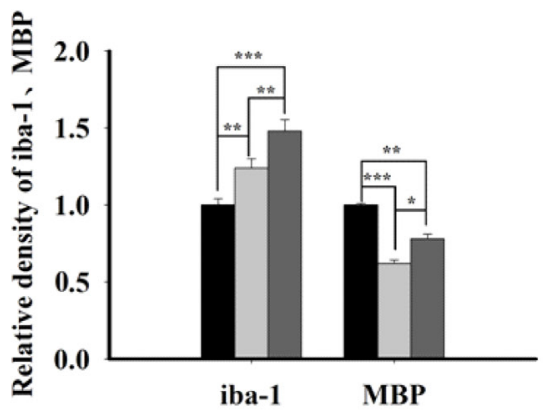

$\mathbf{F}$
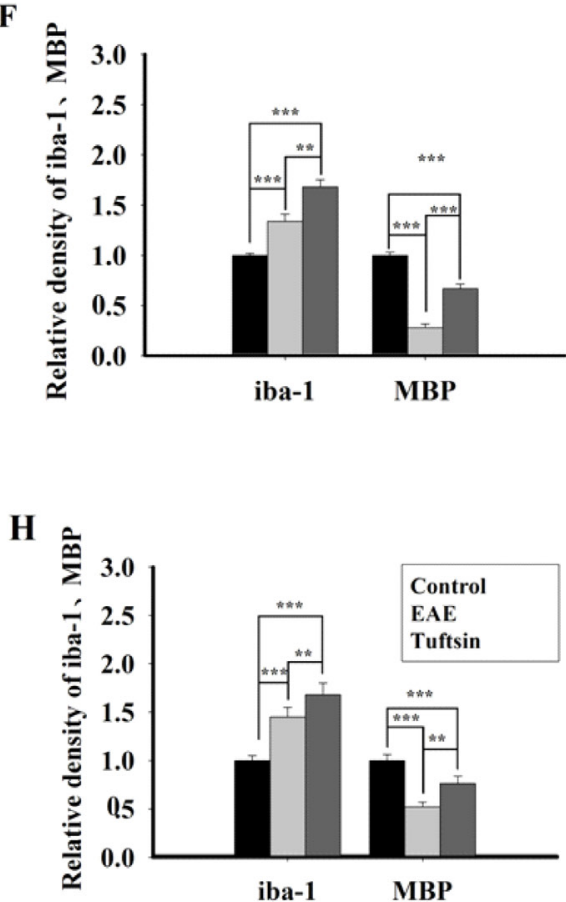

Figure 1. Expression of iba-1 was observed in all three groups. iba-1 and MBP expression of all groups at (A and B) 7 days, (C and D) 14 days, (E and F) 21 days and $(\mathrm{G}$ and $\mathrm{H}) 28$ days. The results are shown as the mean \pm standard error of mean. ${ }^{*} \mathrm{P}<0.05,{ }^{* * *} \mathrm{P}<0.01$ and ${ }^{* * * *} \mathrm{P}<0.001$. iba-1, ionized calcium binding adaptor molecule 1; MBP, myelin basic protein; EAE, experimental autoimmune encephalomyelitis.

the symptoms of EAE in mice. Following induction of EAE with MOG35-55 or PBS, the symptoms of EAE mice with and without tuftsin treatment were compared by using clinical score testing. In Fig. 2A, we observed that in the acute phase of EAE, all groups had similar clinical scores. The clinical scores of the EAE and tuftsin groups changed from day 11; however, the EAE and tuftsin groups achieved a higher clinical score than the control group on day 14, day 21 and day $28(\mathrm{P}<0.05)$. However, during the progression of EAE, the control group had no mice without the disease. Notably, EAE mice treated with tuftsin had a significantly lower clinical score than did mice treated with PBS on day $14(\mathrm{P}<0.05)$, day $21(\mathrm{P}<0.05)$ and day 28 $(\mathrm{P}<0.05)$. These results demonstrated that tuftsin treatment can ameliorate the behavior impairment induced by MOG35-55.

Remyelination was increased when the activation of microglia was stimulated A typical pathological change during EAE development is demyelination, which can be evaluated utilizing LFB staining. To investigate this aspect comprehensively, a histological examination of spinal cord tissue 

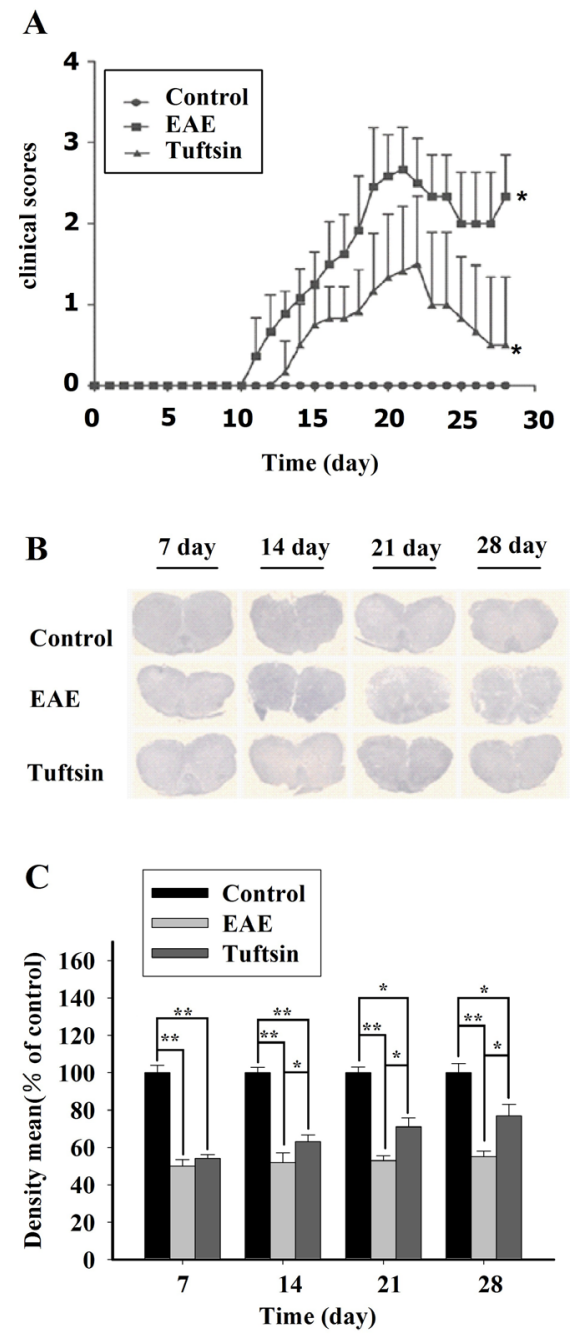

Figure 2. Effects of microglial cells exposed to tuftsin on EAE severity and demyelination. (A) Daily scores were averaged. Control $(n=6)$; EAE $(n=6)$; tuftsin $(\mathrm{n}=6)$ at day 7 , day $14(\mathrm{P}<0.05)$, day $21(\mathrm{P}<0.05)$ and day $28(\mathrm{P}<0.05)$. (B) Demyelination lesions of the spinal cord in the three groups (magnification $\mathrm{x} 40$ ). Luxol fast blue staining at days 7, 14, 21 and 28. (C) Density mean (\% of control). The results are shown as the mean \pm standard error of mean. ${ }^{*} \mathrm{P}<0.05,{ }^{* *} \mathrm{P}<0.01$. EAE, experimental autoimmune encephalomyelitis.

isolated from mice in the control, EAE and tuftsin groups was performed at different time points of the EAE process. The spinal cord tissue sections were subjected to LFB staining in which myelin is stained blue to green, immunohistochemical examination. As shown in Figs. 2B and C, and 3, significant demyelination was not observed in any group on day 7. At day 14, the clinical onset point, LFB staining of the EAE group demonstrated slight demyelination in the white matter. However, in the tuftsin group, the demyelination was less intense at the same time point. The structure of the myelin sheath in the control group, however, exhibited integrity. At the peak of the EAE process, day 21, a gradual but obvious change in the myelin sheath revealed severe deficiency in the EAE group. In contrast, demyelination was milder in the tuftsin group. On day 28, during the recovery stage of EAE, the loss of myelin did not continue but remyelination began, particularly in the tuftsin group, in which remyelination was more obvious than in the EAE group, showing that the structure of the myelin sheath was relatively intact. It should

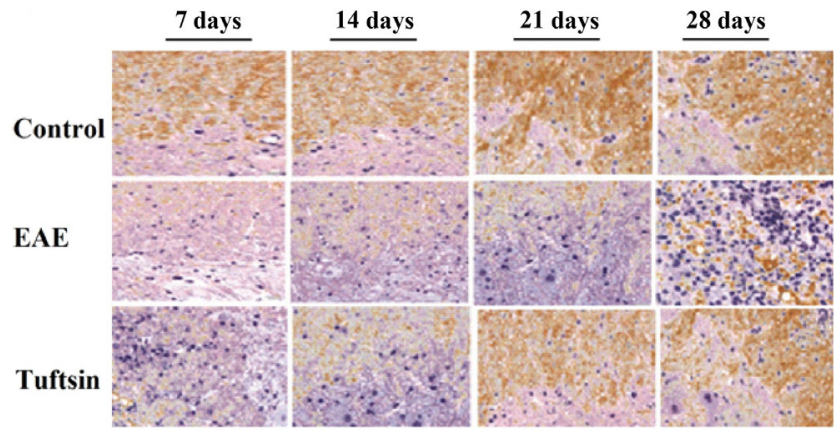

Figure 3. Immunohistochemistry and western blot analyses for the myelin sheath using anti-MBP. Immunohistochemistry for the myelin sheath using MBP in spinal cord sections (magnification, $x 40$ ). The white matter indicated by MBP and the amount of MBP differed at various stages of EAE. MBP, myelin basic protein; EAE, experimental autoimmune encephalomyelitis.

be noted that the structure of myelin in the control group remained unaltered.

Microglia subset alteration alleviates EAE symptoms. Microglia were demonstrated to have M1 and M2 subsets, which represent pro- and anti-inflammatory populations, respectively (12). TNF- $\alpha$ and nitric oxide were secreted by M1 microglia, while neuroprotective M2 microglia produced protective cytokines, including IL-10 and TGF- $\beta$. M2 microglia have been reported to serve a protective role in EAE by ameliorating experimental autoimmune encephalomyelitis (13). To test whether M1 or M2 microglia contributed to the severity of EAE during development, the concentrations of cytokines produced by M1 or M2 microglia were detected. Total RNA was extracted the from the brains from each group and RT-qPCR performed to determine the microglial phenotype based on TNF- $\alpha$ levels for M1, and IL-10 and TGF- $\beta$ levels for M2. Fig. 4 shows that the amounts of TNF- $\alpha$ and TGF- $\beta$ were reduced in the tuftsin group and that the level of IL-10 was increased in the EAE group. The levels of TNF- $\alpha$, IL-10 and TGF- $\beta$ were increased at day 14 in the tuftsin group and EAE group, and the levels of IL-10 and TGF- $\beta$ in the tuftsin-treatment group exceeded those in the EAE group $(\mathrm{P}<0.01$ and $\mathrm{P}<0.05$, respectively), but the level of TNF- $\alpha$ was less than that in the EAE group $(\mathrm{P}<0.01)$. At days 21 and 28, a comparison of the levels of IL-10 and TGF- $\beta$ in the tuftsin treatment group with those in the EAE group revealed that the former increased more than the latter $(\mathrm{P}<0.001, \mathrm{P}<0.001)$; however, the level of TNF- $\alpha$ demonstrated the opposite pattern. The control group generated little TNF- $\alpha$, IL-10 and TGF- $\beta$. Collectively, these data indicated that microglial activation did not contribute to the development of EAE but rather the secretion of protective cytokines, which ameliorated the progression of clinical EAE. Thus, these experiments indicated that microglia released anti-inflammatory cytokines, improving remyelination.

\section{Discussion}

The present study observed the role of microglia in EAE progression and the findings demonstrated that demyelination increased during EAE progression before peaking. By increasing microglial activation, the disease severity of EAE 

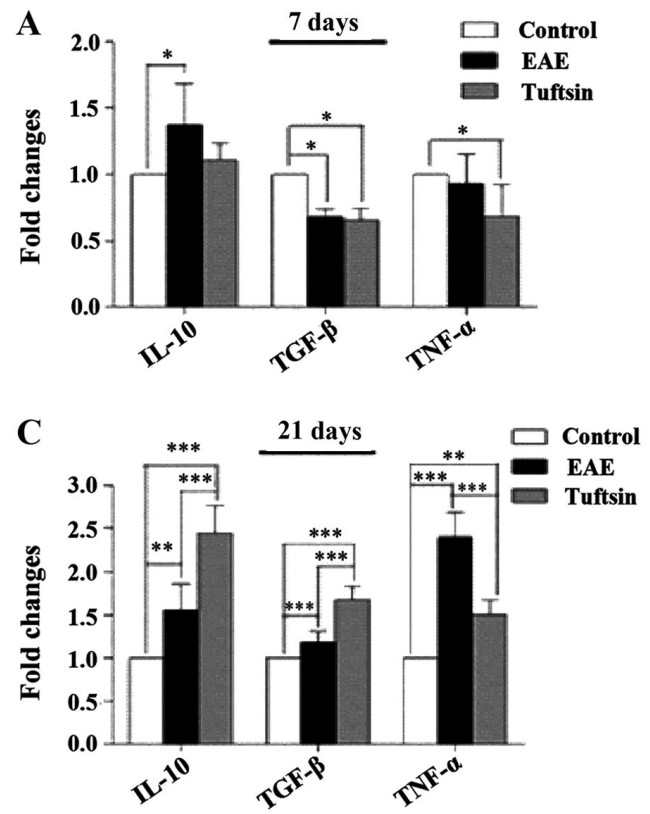
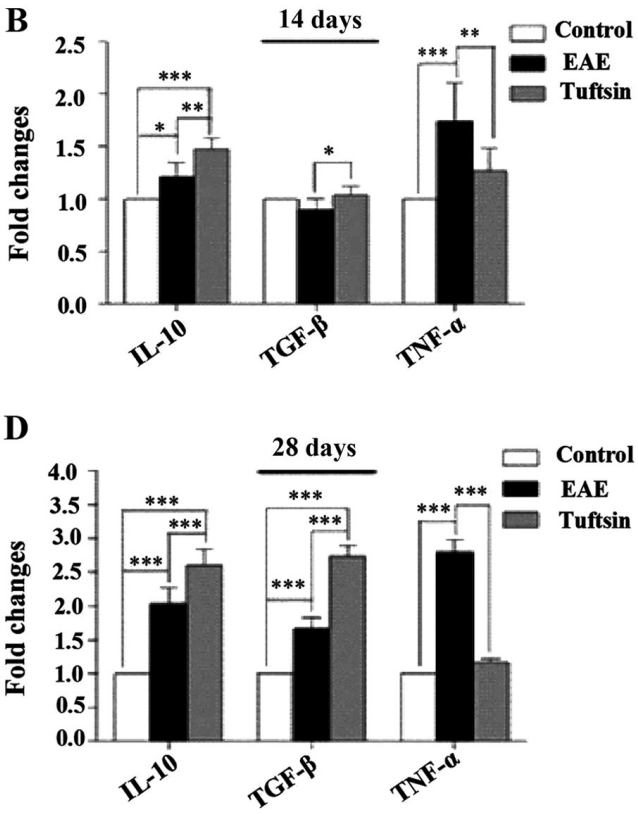

Figure 4. RT-qPCR was performed on RNA from spinal cord homogenates extracted from all three groups at various time points. The proinflammatory cytokine TNF- $\alpha$ and the anti-inflammatory cytokines IL-10 and TGF- $\beta$ produced by microglial cells were quantified at (A) 7, (B) 14 , (C) 21 and (D) 28 days. $\mathrm{n}=6$. The results are shown as the mean \pm standard error of mean. ${ }^{*} \mathrm{P}<0.05,{ }^{* *} \mathrm{P}<0.01$ and ${ }^{* * *} \mathrm{P}<0.001$. RT-qPCR, reverse transcription-quantitative $\mathrm{PCR}$; TNF, tumor necrosis factor; IL, interleukin; TGF, transforming growth factor.

could be suppressed in the chronic phase. However, the recovery of EAE mice occurred earlier in the tuftsin-treated group than in the EAE group, correlating with microglial activation. Generally, activation of microglia reduced EAE severity and advanced the recovery of EAE in C57BL/6 mice.

To investigate the implications of microglia for MS, EAE was induced with MOG35-55 in C57B6/L mice, which is the commonest animal model used to study MS (14). Then, clinical score assessment, a widely accepted behavioral method for evaluating EAE symptoms, was used to observe the variation among the mice. In the present study, mice subjected to MOG35-55 exhibited weakness in the tail and hind limbs. The results of subsequent clinical score testing demonstrated that the mice treated with tuftsin achieved markedly better scores relating to limb weakness and performance compared with their counterparts which did not receive tuftsin, especially during the recovery phase.

Subsequently, the variation of pathology, including LFB staining, was assessed in in the spinal cord because LFB is considered an index of demyelination, which is a widely accepted model for assessing the degree of demyelination at the pathology level (15). LFB staining revealed not only intense mitochondrial staining but also staining for demyelinating fibers both peripherally and in the CNS (16), which was widespread in myelin staining. An increase in myelination in the EAE group was detected compared with the control group and the tuftsin group, which could promote microglia; this effect decreased in the spinal cord compared with the EAE group, especially during the recovery phase. These data indicated that in the recovery phase, microglia could improve remyelination and attenuate the severity of EAE symptoms.

The role of microglia in demyelinating neurodegenerative diseases, MS and its animal model EAE is still controversial (6). Previous data have shown that microglia serve a detrimental role and encompass a wide range of harmful factors, such as serving a toxic role in neurons and oligodendrocyte precursor cells, and releasing proteases, inflammatory cytokines and free radicals (17), as well as recruiting and promoting $\mathrm{T}$ lymphocyte reactivity in the CNS $(18,19)$. Emerging evidence, however, has demonstrated that microglia serve beneficial roles in the brain during neurodegenerative disease (5), particularly in the recovery of EAE progression. The precise mechanisms involved in the protective role of microglia remain unclear, but evidence indicates that microglia serve a vital role, including axonal regeneration, assistance with remyelination, efficient removal of injured myelin debris and release of trophic support factors (6). By phagocytosing cellular debris (20) and releasing trophic support factors and anti-inflammatory cytokines, microglia can protect neurons from injury and repair the injured brain and spinal cord (21). Furthermore, all of these effects contribute to myelin regeneration in MS and EAE, in which remyelination occurs spontaneously (22).

One mechanism of remyelination is the efficient clearance of myelin debris that contains inhibitory factors for axonal growth, which is a critical step during the remyelination progress (23). Microglia, the resident immune cells in the CNS, could serve a phagocytic role similar to that of other cells of monocytic origin. Microglial phagocytosis has been suggested to remove myelin debris and apoptotic cell debris during demyelination and is beneficial to promoting regeneration (21). Independent studies have shown that microglia function through lectin-, integrin-, and phosphatidylserine-mediated recognition of apoptotic neurons, followed by phagocytosis and clearance of apoptotic neurons (24). In the present study, administering tuftsin promoted phagocytosis for microglia expressing tuftsin receptors and enhanced the phagocytosis of microglia. Therefore, tuftsin treatment may upregulate the role 
of phagocytosis by combining with its receptor on microglia, which could efficiently clear the debris from disrupted myelin sheaths and injured neurons and may maintain an environment favorable for remyelination.

One important factor in remyelination is oligodendrocyte precursor cells (25), which migrate to the demyelination lesion, differentiate into myelinating oligodendrocytes and synthesize myelin (26). A previous study demonstrated that remyelination occurs during demyelination progression, in which endogenous oligodendrocyte precursor cells (OPCs) intend to remyelinate, but this process fails (25). In addition, blocking the differentiation of oligodendroglial progenitor cells causes remyelination failure during MS progression (27). A study indicated that different states of microglia were related to oligodendrogenesis by producing IGF-1 and downregulating TNF- $\alpha$ (28). Microglia have been demonstrated to present a cytokine and chemokine repertoire, which enables them to activate and recruit endogenous OPCs to the MS lesion and transmit trophic support during remyelination.

Inflammatory autoimmune attack against myelin in the CNS leads to myelin destruction and demyelinated lesion formation (plaques) in MS (29). Thus, inhibition of the inflammatory response, instead of improving anti-inflammation, contributes to remyelination. Microglia serve an important role in the pathology and pathogenesis of MS. A previous study suggested that microglia might express immune receptors, nod-like receptors (NLRs), which are intracellular receptors capable of suppressing inflammation and ameliorating EAE symptoms (30). In addition, deregulation of cytokines (M1 vs. M2) has been reported to be involved in the pathogenesis of remyelination (31). An previous study demonstrated that microglia/macrophages contribute to driving oligodendrocyte differentiation during CNS remyelination by shifting the phenotype from proinflammatory M1 to anti-inflammatory M2, which triggers remyelination (32). The $\mathrm{M} 2$ phenotype of microglia secrete IL-10 and TGF- $\beta$, providing evidence for the induction of an anti-inflammatory M2 phenotype to suppress innate immunity in affected brain tissue (31). TGF- $\beta$ is a signature of microglia and exerts a protective effect against various neuronal insults (33). Therefore, the M1/M2 balance in EAE was determined by measuring TNF- $\alpha$ as an M1 cytokine and IL-10 and TGF- $\beta$ as M2 cytokines. In the present study, EAE induced a decrease in IL-10 and TGF- $\beta$ but an increase in TNF- $\alpha$ in the spinal cord; however, tuftsin treatment normalized these cytokine levels. On the other hand, a previous study indicated that inflammation can convert a non-remyelinating context to a remyelinating context (34).

Together, the results the present study illustrated the possibility that microglia may be activated towards an anti-inflammatory state by shifting to the M2 mode, generating a properly regulated immunosuppressive response. The findings indicated that microglial activation could significantly enhance remyelination induced by anti-immunization in mice with EAE. Diminishing the response to inflammation and enhancing white matter lesions may result from the neuroprotective effect of microglia. More studies are required to detail the mechanisms of microglial activation at the molecular level by ameliorating neurologic deficits. The results of the present study provide useful information for considering the intervention of microglial activation as an alternative treatment choice for multiple sclerosis.

As for the concentration of the tuftsin, it was thought to be the optimal concentration. At this concentration, there were very few adverse reactions and no mortality in the animals, and this concentration has a good therapeutic effect. However, there were two animals which appeared to be in intolerable distress and self-mutilated limbs and culled for this reason. Of course, in future experiments, we will continue to explore the concentration of the tuftsin to find the more appropriate concentration.

\section{Acknowledgements}

Not applicable.

\section{Funding}

No funding was received.

\section{Availability of data and materials}

The datasets used and/or analyzed during the current study are available from the corresponding author on reasonable request.

\section{Authors' contributions}

$\mathrm{ZB}$ and FF designed the research study. YL performed the experiments and research. $\mathrm{JH}$ analyzed the data and wrote the manuscript. All authors contributed to editorial changes in the manuscript. All authors read and approved the final manuscript.

\section{Ethics approval and consent to participate}

All protocols were approved by the Animal Ethics Committee of the North China University of Science and Technology, and animal handling was carried out according to the guidelines from the National Institutes of Health.

\section{Patient consent for publication}

Not applicable.

\section{Competing interests}

The authors declare that they have no competing interests.

\section{References}

1. Xu L, He D and Bai Y: Microglia-mediated inflammation and neurodegenerative disease. Mol Neurobiol 53: 6709-6715, 2016.

2. Kreutzberg GW: Microglia: A sensor for pathological events in the CNS. Trends Neurosci 19: 312-318, 1996.

3. Suzumura A: Neurotoxicity by microglia: The mechanisms and potential therapeutic strategy. Fukuoka Igaku Zasshi 100: 243-247, 2009 (In Japanese).

4. Kim SU and de Vellis J: Microglia in health and disease. J Neurosci Res 81: 302-313, 2005. 
5. Rawji KS and Yong VW: The benefits and detriments of macrophages/microglia in models of multiple sclerosis. Clin Dev Immunol 2013: 948976, 2013.

6. Napoli I and Neumann H: Protective effects of microglia in multiple sclerosis. Exp Neurol 225: 24-28, 2010.

7. Rus H, Cudrici C, Niculescu F and Shin ML: Complement activation in autoimmune demyelination: Dual role in neuroinflammation and neuroprotection. J Neuroimmunol 180: 9-16, 2006.

8. Fridkin $M$ and Najjar VA: Tuftsin its chemistry, biology, and clinical potential. Crit Rev Biochem Mol Biol 24: 1-40, 1989.

9. Bump NJ and Najjar VA: Tuftsin (Thr-Lys-Pro-Arg), a natural modulator of macrophage activity: Further studies. Mol Cell Biochem 63: 137-142, 1984

10. Livak KJ and Schmittgen TD: Analysis of relative gene expression data using real-time quantitative PCR and the 2(-Delta Delta C(T)) method. Methods 25: 402-408, 2001.

11. Terry RL, Ifergan I and Miller SD: Experimental autoimmune encephalomyelitis in mice. Methods Mol Biol 1304: 145-160, 2016.

12. Kigerl KA, Gensel JC, Ankeny DP, Alexander JK, Donnelly DJ and Popovich PG: Identification of two distinct macrophage subsets with divergent effects causingeither neurotoxicity or regeneration in the injured mouse spinal cord. J Neurosci 29 $13435-13444,2009$

13. Wu M, Nissen JC, Chen EI and Tsirka SE: Tuftsin promotes an Anti-Inflammatory switch and attenuates symptoms in experimental autoimmune encephalomyelitis. PLoS One 7: e34933, 2012.

14. Baker D and Amor S: Experimental autoimmune encephalomyelitis is a good model of multiple sclerosis if used wisely. Mul Scler Relat Disord 3: 555-564, 2014

15. Salthouse TN: Luxol fast blue $G$ as a myelin stain. Stain Technol 39: 123, 1964.

16. Snodgress AB, Dorsey $\mathrm{CH}$ and Lacey LB: Luxol fast blue staining of degenerating myelinated fibers. Anat Rec 140: 83-90, 1961.

17. Benveniste EN: Role of macrophages/microglia in multiple sclerosis and experimental allergic encephalomyelitis. J Mol Med (Berl) 75: 165-173, 1997.

18. Bilimoria PM and Stevens B: Microglia function during brain development: New insights from animal models. Brain Res 1617: 7-17, 2015.

19. Rothhammer V and Quintana FJ: Role of astrocytes and microglia in central nervous system inflammation Introduction Semin Immunopathol 37: 575-576, 2015.

20. Lampron A, Larochelle A, Laflamme N, Préfontaine P, Plante MM, Sánchez MG, Yong VW, Stys PK, Tremblay MÈ and Rivest S: Inefficient clearance of myelin debris by microglia impairs remyelinating processes. J Exp Med 212: 481-495, 2015.

21. Program and Abstracts of the 5th Joint Italian-German Purine Club Meeting: 'Fostering translational research on Purines by Italian-German joint efforts'. Purinergic Signal 10: 369-417, 2014.

22. Piaton G, Williams A, Seilhean D and Lubetzki C: Remyelination in multiple sclerosis. Prog Brain Res 175: 453-464, 2009.
23. Zhang L, Johnson D and Johnson JA: Deletion of Nrf2 impairs functional recovery, reduces clearance of myelin debris and decreases axonal remyelination after peripheral nerve injury. Neurobiol Dis 54: 329-338, 2013.

24. Witting A, Muller P, Herrmann A, Kettenmann H and Nolte C: Phagocytic clearance of apoptotic neurons by microglia/brain macrophages in vitro: Involvement of lectin-, integrin-, and phosphatidylserine-mediated recognition. J Neurochem 75 1060-1070, 2000

25. Bramow S, Frischer JM, Lassmann H, Koch-Henriksen N Lucchinetti CF, Sørensen PS and Laursen H: Demyelination versus remyelination in progressive multiple sclerosis. Brain 133: 2983-2998, 2010.

26. Tanaka T and Yoshida S: Mechanisms of remyelination: Recent insight from experimental models. Biomol Concepts 5: 289-298, 2014.

27. Kuhlmann T, Miron V, Cuo Q, Wegner C, Antel J and Brück W: Differentiation block of oligodendroglial progenitor cells as a cause for remyelination failure in chronic multiple sclerosis. Brain 131: 1749-1758, 2008

28. Butovsky O, Landa G, Kunis G, Ziv Y, Avidan H, Greenberg N, Schwartz A, Smirnov I, Pollack A, Jung S and Schwartz M: Induction and blockage of oligodendrogenesis by differently activated microglia in an animal model of multiple sclerosis. J Clin Invest 116: 905-915, 2006.

29. Dutta R and Trapp BD: Mechanisms of neuronal dysfunction and degeneration in multiple sclerosis. Prog Neurobiol 93: 1-12, 2011.

30. Gharagozloo M, Mahvelati TM, Imbeault E, Gris P, Zerif E, Bobbala D, Ilangumaran S, Amrani A and Gris D: The nod-like receptor, Nlrp12, serves an anti-inflammatory role in experimental autoimmune encephalomyelitis. J Neuroinflamm 12: 198 , 2005.

31. Mikita J, Dubourdieu-Cassagno N, Deloire MS, Vekris A, Biran M, Raffard G, Brochet B, Canron MH, Franconi JM, Boiziau C and Petry KG: Altered M1/M2 activation patterns of monocytes in severe relapsing experimental rat model of multiple sclerosis. Amelioration of clinical status by M2 activated monocyte administration. Mult Scler 17: 2-15, 2011.

32. Miron VE, Boyd A, Zhao JW, Yuen TJ, Ruckh JM, Shadrach JL, van Wijngaarden P, Wagers AJ, Williams A, Franklin RJM and Ffrench-Constant C: M2 microglia and macrophages drive oligodendrocyte differentiation during CNS remyelination. Nat Neurosci 16: 1211-1275, 2013

33. Butovsky O, Jedrychowski MP, Moore CS, Cialic R, Lanser AJ, Gabriely G, Koeglsperger T, Dake B, Wu PM, Doykan CE, et al: Identification of a unique TGF- $\beta$ dependent molecular and functional signature in microglia. Nat Neurosci 17: 131-143, 2014.

34. Foote AK and Blakemore WF: Inflammation stimulates remyelination in areas of chronic demyelination. Brain 128: 528-539, 2005.

(7) $\mathrm{E}$ This work is licensed under a Creative Commons Attribution-NonCommercial-NoDerivatives 4.0 International (CC BY-NC-ND 4.0) License. 